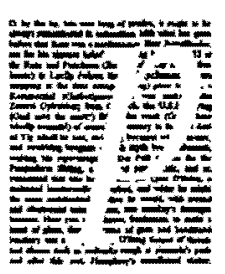

\title{
Lo real-maravilloso y la tradición europea: La utopía como catalizador discursivo en los textos de Cristóbal Colón, Paul Gauguin y Alejo Carpentier
}

\author{
Míchael Schuessler \\ Universidad de California, Los Ángeles
}

$\mathrm{E}^{\mathrm{n}}$ n este breve ensayo me propongo estudiar una faceta de la génesis literaria de lo real-maravilloso según fue concebido y desarrollado por Alejo Carpentier. ।

Para llegar a una comprensión más profunda de los orígenes de su proyecto literario, es útil explorar la relación entre los dos periodos históricos que más influencia ejercieron sobre el desarrollo literario del novelista cubano: el re'nacimiento y la edad moderna. Aunque lejanos en términos cronológicos, estos dos momentos están íntimamente relacionados en su utilización del concepto o la posibilidad de la utopía como un catalizador del discurso creativo. ${ }^{2}$ Dos ejemplos de este fenómeno-el «descubrimiento» de América por Cristóbal Colón en 1492 y el re-descubrimiento de las culturas «primitivas» de Oceanía en 1891 por Paul Gauguin--constituyen las dos manifestaciones culturales de estos periodos históricos que sustentan el enfoque de este estudio. ${ }^{3}$ Para demostrar esta conexión será necesario analizar varios textos representativos, en un intelito de ilustrar el trasfondo histórico-filosófico que produce lo que va a constituir 10 real-maravilloso americano.

Una muestra de la influencia de estos dos periodos históricos en ]a obra de Carpentier se encuentra'plenamente desarrollada en lo que Carlos Fuentes denomina «la búsqueda de la utopía;» tema fundamental de Los pasos perdidos $\underline{\text { (Fuentes } 122-48)}$. Sin embargo, es en el prólogo a El $\underline{\text { El reino }} \underline{\text { de }} \underline{\text { este }} \underline{\text { mundo donde }}$ 
Carpentier manifiesta por primera vez lo que va a constituir su proyecto literario hasta el final de su vida. Este «manifiesto» de lo real-maravilloso ilustra la profunda huella que dejan en Carpentier los proyectos ideológicos de dos periodos históricos que, en este trabajo, serán ilustrados por medio de una lectura de los escritos de Cristóbal Colón y Paul Gauguin, ambos descubridores, como veremos, de una anhelada utopía ultramarina. ${ }^{5}$

Estos dos periodos, que se desarrollaron a finales del siglo XV y XIX, respectivamente, tienen como vínculo los problemas derivados de una crisis existencial incorporada en la necesidad de resucitar las realidades humanas perdidas por la deshumanización del mundo contemporáneo. ${ }^{6}$ Esta crisis, desde luego, no se limita a la esfera de las ciencias sociales, sino que aparece también en las expresiones humanas contemporáneas tanto, plásticas como literarias. De modo que la propuesta literaria carpentieriana será considerada en función de una malaise creativa occidental que envolvía la comunidad artístico-intelectual desde los finales del siglo pasado, la cual engendró un renovado interés en las culturas «primitivas»-vestigios de la colonización mundial iniciada por los anhelos expansionistas del siglo XV e incorporados en los viajes de Cristóbal Colón. Es precisamente la búsqueda de una utopía humana en la forma de estas culturas primordiales (y, por lo tanto, incorruptas) lo que caracteriza tanto el descubrimiento de América como su re-descubrimiento a finales del siglo XIX y principios del veinte.

Debido a estos factores, 'se analizará el proyecto literario de Carpentier en términos de su problemática ideol6gica, producto de sus raíces europeas que han fomentado una interpretación eurocéntrica de un movimiento literario cuyo objetivo ha sido denominado la recuperación de una voz literaria latinoamericana. ${ }^{7}$ Hasta cierto punto se puede demostrar que con lo real-maravilloso Carpentier continúa la tradición iniciada por Colón y renovada en la Europa del siglo XIX, con el propósito de resucitar el ambiente cultural europeo. Sin embargo, en el caso del autor cubano, esta visión es proyectada hacia América en el intento de recuperar una tradición propia y alejarse de la imitación servil de los extenuados modelos europeos de la representación artística. Por lo tanto, lo que propone Carpentier es, esencialmente, el resultado de una necesidad europea de auto-definición a través del «otro» en un intento de rescatar los valores humanos de una cultura rápidamente enajenada por la mecanización del hombre. ${ }^{8}$ En este sentido, se puede demostrar que Carpentier no propone nada original en cuanto al desarrollo de una literatura latinoamericana al definirla-como siempre había sido definida hasta ese momento-por contraste con Europa. En otras palabras, lo real-maravilloso como proyecto literario latinoamericano surge del ambiente europeo contemporáneo y por consiguiente no tendría ninguna realización (ni 
necesidad de ser realizado) si no fuera visto y definido a través del ojo europeo. En este sentido la literatura latinoamericana constituye lo que Edmundo O'Gorman ha denominado un ens ab alio por el hecho de que América y, por asociación su literatura, debe su existencia a una entelequia cultural, Europa Es uno de los propósitos de esta investigación cuestionar la posibilidad de no ver a América sino por contraste de Europa y notar cómo, en el caso de Carpentier. es precisamente la simbiosis cultural que engendró el «descubrimiento» de América lo que crea un aspecto de «lo real-maravilloso» carpentieriano; hecho que lo distingue de otros proyectos artísticos del mismo periodo ya que éste intenta rescatar de una manera consciente una tradición netamente latinoamericana.

Para emprender este análisis es relevante cotejar la visión del mundo «primitivos como interpretado por el hombre del siglo $\mathrm{X} 1 \mathrm{X}-\mathrm{XX}$ con la de él quien en 1492 lo «descubrió.» Como ya se sabe, una visión utópica está claramente expuesta en muchas descripciones que Cristóbal Colón hace de las islas que explora durante su primer viaje a las Indias. En la «Carta de Cristóbal Colón al escribano de ración Luis de Sant' Angel,» el primer documento escrito sobre lo que sería bautizado como Nuevo Mundo, Colón, convencido de estar en las periferias de la gran civilización de Cipangu, observa que, geográficamente, en contraste con el mundo europeo, «todas las otras [islas] son fertilísimas en demasiado grado ... En ella, ay muchos puertos en la costa de la mar, sin comparacion de otros que yo sepa en Cristianos, y fartos mas, y buenos y grandes, que es maravilla.» (The Spanish Letter 2). De los habitantes de esta. tierra maravillosa explica. que:

Verdad es, que después que aseguran, y pierden este mièdo, ellos son tanto sin engaño y tan liberales de lo que tienen, que no lo creer(an sino el que lo viese. Ellos de cosa que tengan, pidiéndosela, jamás dizen de nó; antes convidan la persona con ello, y muestran tanto amor que darian los corazones; y quier sea cosa de valor, quier sea de poco precio, luego por qualquiera cosica, de qualquiera manera que sea, que seles de por ello, sean contentos. (3)

En esta primera descripción ya notamos una serie de características que sirven para separar a los habitantes de estas islas de los del mundo europeo. Esto por el hecho de que ellos son buenos en la misma medida que los europeos son malos; es decir son--en ténninos morales-lo que no son pero deben ser los europeos contemporáneos. ${ }^{9}$ En vez de ser conservadores son liberales, en vez de ser avaros, son generosos y en vez de ser rencorosos, son amorosos. De esta manera incorporan un espejo moral que Colón utiliza para ver hasta qué punto se ha. degenerado su propia cultura.. 
Al describir el medio ambiente de las islas recién exploradas, notamos una representación que se aproxima a la del paraíso terrenal. En el cuaderno de bitácora del primer viaje, por ejemplo, encontramos en el día 21 de octubre la siguiente descripción:

aquíy en toda la isla son todos verdes y las yervas como en el abril en el Andaluzía. Y el cantar de Los paxaritos que parecè qu'el hombre nunca se querría partir de aquí, y las manadas de los papagayos ascurecen el sol, y aves y paxaritos de tantas maneras y tan diversas de las nuestras que es maravilla. y después ha árboles de mili maneras y todos dan de su manerafruto, y todos guelen qu'es maravilla... (77)

Aquí se resaltan tres elementos clave de la descripción geográfica del almi. rante durante su primer viaje: en primer lugar el fervor de recrear el paisaje maravilloso en toda su abundancia exótica que, en este ejemplo, incluye el cantar de los pájaros y la fragancia de los árboles. Si no fuera por un elemento foráneo (el «papagayo»), esta descripción podría sustituir el Locus amæenus tan anhelado por los poetas de la España medieval. Otra vez, este ambiente está descrito en ténninos positivos que ilustran lo negativo del Viejo Mundo, o por lo menos, la presencia constante de lo mejor que tiene el mundo conocido (en este caso el clima y la naturaleza);

Las actitudes que sirven para vincular el pensamiento de Colón a la desesperación de la edad moderna tambIén se encuentran en sus descripciones de la gente en términos de lo que no tienen y cómo no se asemejan a los europeos y, por lo tanto, según el almirante, son mejores. Un mes después del hallazgo de tierra Colón describe los habitantes de esta manera:

porque yo vi e cognozco. .. qu'esta gente no tiene secta ninguna ni son
idólatras, salvo muy mansos y sin saber que sea mal ni matar a otros
ni prender, y sin armas y tan temerosos que a una persona de los
nuestros iuyen ciento d'ellos. aunque burlen con ellos, y crédulos y
cognoscedores que ay Dios en el cielo, efirmes que nosotros avernos
venido del cielo ... (94)

Si escrutamos los textos del almirante en ténninos de lo que implican sus descripciones de las tierras «descubiertas,» podemos asumir que en la Europa de su epoca existían sectas heréticas, los hombres no eran «mansos» sino seres belicosos y asesinos y, peor aún, mientras los indios eran crédulos, los europeos eran ateos. ${ }^{10}$ De modo que, basándose en estas descripciones, se puede afirmar que Colón ve a este mundo desconocido como repositorio de los valores humanos perdidos por la decadencia de la Europea contemporánea con sus constantes 
guerras, injusticias y maldades. La idealización de estas tierras eventualmente llega a tal grado que en la «Relación del cuarto viaje» (1503) Col6n insiste en que parte de ellas constituye el Paraíso Terrenal: «El mundo es poco; el injuto d'ello es seis partes, la séptima solamente cubierta de agua. La experiencia ya está vista, y la escriví por otras letras y con adornamiento de la Sacra Escritura con el sitio del Paraíso Terrenal que la Sancta Iglesia aprueva» (283).

En una actitud muy parecida a la del explorador renacentista, existía en varios grupos de individuos del fin de siécie europeo el mismo deseo de encontrar en las culturas «primitivas» la magia primordial extraviada para siempre de las zonas urbanas e industrializadas del Viejo Mundo, junto con los mitos, las leyendas y la realidad extraordinaria propia de tierras tan alejadas del progreso tecnoíógico del mundo «civilizado.»11 Como en el Renacimiento, el redescubrimiento de las culturas asiáticas, africanas, oceánicas y americanas surgió de una necesidad de reinstalar en el mundo conocido nuevas formas, expresiones y realidades humanas. ${ }^{12}$ Parto de la premisa que un aspecto de las raíces ideolÓgicas y estéticas del proyecto literario de Carpentier se encuentra en un grupo de artistas que anticiparon el movimiento surrealista, los cuales podemos ubicar bajo el rubro de «primitivistas.»

La creación de museos enciclopédicos, los avances en comunicación y el anhelo de encontrar algo verdadero y humano en un mundo cicatrizado por la industrialización y enajenación del mundo contemporáneo fueron factores que promovieron el re-descubrimiento de un mundo desconocido, olvidado, misterioso, maravilloso. Otra vez Asia, África y América entraron en la conciencia occidental como la posibilidad de una utopía perdida dentro de la modernidad deshumanizadora. Irónicamente, la creación de espacios públicos que permitían el contacto con el mundo «primitivon constituyó un producto del mismo mundo «civilizado» tan despreciado por algunos artistas de este periodo. El ejemplo más citado de este fenómeno es el de la Feria Mundial celebrada en París en 1889 que, aparte de exponer los logros tecnológicos de la época, incluyó también muestras de culturas «primitivas,» muchas de las cuales procedentes de las colonias ultramarinas del mundo «civilizado» (Hughes 9).

Es útil señalar aquí la influencia inmediata que tuvo el arte primitivo en el desarrollo del arte moderno europeo. La obra de Pablo Picasso Les demoiselles d'Avignon (1907) que, según muchos historiadores del arte, inicia la pintura moderna europea, al mismo tiempo demuestra una profunda influencia «primitiva» en la forma de máscaras africanas y caras ibéricas con que nos miran las figuras del cuadro. ${ }^{13}$ Se puede afirmar, por lo tanto, que la influencia de las creaciones--con su abstracción geométrica y mitos explotables-fabricadas por culturas marginadas del mundo industrializado, permea el mundo artístico euro- 
pea y determina, hasta cierto punto, su desarrollo estético. Para demostrar esto sólo hay que pensar en movimientos como el Cubismo en Francia y el Expresionism() en Alemania, que son herederos directos de esta tendencia. No es de sorprender, entonces, que lo contrario-Ia imposición y consiguiente simbiosis de la tradición occidental en las culturas primitivas (en este caso las de América)-haya suscitado una ideología tan determinante para un escritor latinoamericano como Carpentier.

El pintor francés de origen mestizo (peruano) Paul Gauguin, quien servía como modelo anterior para los miembros del grupo surrealista, había forjado a mediados del siglo XIX una filosofía artística basada en el vanguardismo de su época (el simbolismo) antes de abandonar la decadente cultura europea para salir a Oceanía. Huye de Francia para viajar a Tahití porque, en sus palabras:

A terrible epoch is brewing in Europe for the coming generation: the kingdom ofgold. Everything is putrefted, even men, even the arts. There [in Tahiti], at least, under an etemally summer sky on a marvellously fertile soil, the Tahitian has only to lift his hands 10 gather his food; and in addition he never works. When in Europe men and women survive only after unceasing labor in which they struggle in convulsions of eoldand hunger, a p; ey lo misery, the Tahitians, on the contrary, happy inhabilanls of the unknown paradise ofOceania, know only Ihe sweetness oflife. ${ }^{14}$ (Chipp 79)

Si comparamos este párrafo con lo previamente citado de los textos colom. binos, inmediatamente. notamos una secuencia de afinidades en términos de la descripción de esta gente y su medio ambiente. Aparte de vivir en lo que parece ser un paraíso terrenal donde bajo el benéfico sol no hay que trabajar para sobrevivir, los habitantes están fuera del peligro de las atrocidades contemporáneas del mundo «civilizado,» ya que solo conocen «the sweetness of life.»En una articulación más franca que la de Colón, Gauguin señala la decadencia de su cultura (y el futuro apocalíptico de ésta) por medio de una visi6n ut6pica del mundo «primitivo.» Si este ejemplo no fuera suficiente para demostrar la afinidad entre las interpretaciones de Col6n y Gauguin, s610 hay que volver al texto colombino para encontrar una referencia que parece directamente reproducida en la cita de Gauguin. Al describir las plantas comestibles de una isla, Colón se maravilla de la abundancia de vegetaci6n sin la necesidad de cultivo humano. Señala que:

tienenfaxones y favas muy diversas de las nuestras, y mucho algod6n, el cual no siembran y nace por los montes árboles grandes, y creo que en todo tiempo lo aya para coger, porque vi los congujQs abiertos y 
otros que se abrtan y flores, todo en un árbol, y otras mill maneras de frutas que me no es posible escrevir, y todo deve de ser cosa pro: vechosa. (90)

Ya que hemos ilustrado varias afinidades entre los textos de Col6n y Gauguin, destacando su visi6n utópica del mundo ultramarino, ahora es necesario vincular esta tradici6n con el proyecto literario de Carpentier. Afortunadamente, su novela Los pasos perdidos proporciona una visión del mundo «primitivo» muy parecida a las descritas por Col6n y Gauguin. S610 al considerar el argumento de la novela de Carpentier podemos notar muchas similitudes. Se trata de un hombre, arrancado de su lugar de origen (Cuba) que, desilusionado por la enajenaci6n del mundo «civilizado» de Estados Unidos, emprende un viaje al «vasto pars'de las utopías permitidas, de los Icarias posibles;» es decir, América del Sur, precisamente Venezuela (391). Como los de Col6n y Gauguin, este viaje se emprende por la necesidad de resucitar los valores espirituales perdidos en el mundo moderno, ya que, según el narrador: «los discursos habían sustituido a los mitos, las consignas, a las dogmas. Hastiado del lugar común fundido en hierro, del texto expurgado y de la cátedra desierta, me acerqué nuevamente al atlántico con el ánimo de pasarlo ahora en sentido inverso» (217). En muchos sentidos su experiencia en el,Nuevo Mundo repite la del cronista de Indias, en este caso la de Fray Servando Castillejos. Al leer su crónica se da cuenta de que:

la añeja prosa sigue válida. Donde el autor señalaba una piedra con perfil de saurio, erguida en la orilla derecha, he visto la piedra con perfil de saurio, erguida en la orilla derecha. Donde el cronista se asombraba ante la presencia de árboles gigantes, he visto árboles gigantescos, hijos de aquellos, nacidos en el mismo lugar, habitados por los mismos pájaros, fulminados por los mismos rayos. (237-8)

Lejos de rechazar a los habitantes del Nuevo Mundo, el narrador se $<<$ pregunta ya si el papel de estas tierras en la historia humana no sería el de hacer posibles, por vez primera, ciertas simbiosis de culturas ... » (247) las cuales, comodespués se verá, son precisamente las que sirven de cimiento para el proyecto literario del. novelista cubano. Al enfrentarse con la realidad increíble de aquel mundo desconocido, el narrador de Los pasos perdidos, como un nuevo Colón, admite que:

tenta mi memoria que irse al mundo del Basca, a las Babeles imaginarias de los pintores de lo fantástico, de los más alucinados ilus. tradores de tentaciones de santos, para hallar algo semejante a lo que estaba contemplando. Y aun cuando encontraba una analogía, tenía 
que renunciar a ella, al punto, por un (sic) cuestión de proporciones. (303)

Como el almirante del mar océano, el narrador de la novela de Carpentier no puede experimentar la maravillosa realidad del Nuevo Mundo sino a través de un lente (en este caso artístico) europeo. En ambos casos, lo visto en América, aunque incorpore un fenómeno directamente experimentado, constituye una revelación fantástica que va más allá de la realidad conocida.

Esencialmente, es en su descripción utópica de la gente que habitaestas selvas tupidas donde se encuentra la visión que une las experiencias del narrador de Los pasos perdidos a las de Colón y Gauguin. El narrador de Los pasos perdidos nos dice que:

esos individuos con piernas y brazos que veo ahora, tan semejantes a mí; esas mujeres cuyos senos son ubres fláccidas que cuelgan sobre vientres hinchados; esos niños que se estiran y ovillan con gestos felinos,' esas gentes que aún no han cobrado el pudor primordial de ocultar los órganos de la generación, que están desnudas sin saberlo, como Adán y Eva antes del pecado, son hombres, sin embargo. No han pensado todavía en valerse de la energía de la semilla; no se han asentado, ni se imaginan en acto de sembrar; andan delante de sí, sin rumbo, comiendo corazones de palmeras, que van a disputar con los simios, allá arriba, colgándose de las techumbres de la selva. (313)

En vez de ser una experienCia negativa, la del narrador cobra un valor trascendente ya que él (como Gauguin) ha «encontmdo en todas partes la solicitación inteligente, el motivo de meditación, formas de arte, de poesía, mitos, más in.structivos para comprender al hombre que cientos de libros escritos en las bibliotecas por hombres jactanciosos de conocer al Hombre» (341).

Al relacionar'los textos que hemos visto con el proyecto de Carpentier es preciso cuestionar la originalidad del mismo en la medida que recupera o no una «tradición» latinoamericana 15 Algunas de las teorías desarrolladas en el libro La invención de América de Edmundo O' Gorman proporcionarán la estructura para esta discusión, la cual me permitirá explorar más profundamente lo original de Carpentier.

Si bien durante los siglos XV YXVI la realidad de la «cuarta orbis pars»--vacía en términos significalivos-se prestaba para su invención europea, en los siglos XIX Y XX, es el producto ideológico de esta misma creación el que genera el proyecto carpentieriano. Mientras que los artistas europeos se apegaron a las expresiones exóticas de lo poco que quedaba de un paraíso perdido, irremediablemente desaparecido, Carpentier logró hallarlo en las expresiones sincréticas que 
que renunciar a ella, al punto, por un (sic) cuestión de proporciones. (303)

Como el almirante del mar océano, el narrador de la novela de Carpentier no puede experimentar la maravillosa realidad del Nuevo Mundo sino a través de un lente (en este caso artístico) europeo. En ambos casos, lo visto en América, aunque incorpore un fen6meno directamente experimentado, constituye una revelación fantástica que va más allá de la realidad conocida.

Esencialmente, es en su des ¿ripción ut6pica de la gente que habita estas selvas tupidas donde se encuentra la visión que une las experiencias del narrador de Los pasos perdidos a las de Colón y Gauguin. El narrador de Los pasos perdidos nos dice que:

esos individuos con piernas y brazos que veo ahora, tan semejantes a mf; esas mujeres cuyos senos son ubres fláccidas que cuelgan sobre vientres hinchados; esos niños que se estiran y ovillan con gestos felinos; esas gentes que aún no han cobrado el pudor primordial de ocultar los órganos de la generación, que están desnudas sin saberlo, como Adán y Eva antes del pecado, son hombres, sin embargo. No han pensado rodavfa en valerse de la energfa de la semilla; no se han asentado, ni se imaginan en acto de sembrar; andan delante de sí, sin rumbo, comiendo corazones de palmeras, que van a disputar con los simios, allá arriba, colgándose de las techumbres de la selva. (313)

En vez de ser una experiencia negativa, la del narrador cobra un valor trascendente ya que él (como. Gauguin) ha «encontrado en todas partes la solicitación inteligente, el motivo de meditaci6n, formas de arte, de poesía, mitos, más instructivos para comprender al hombre que cientos de libros escritos en las bibliotecas por hombres jactanciosos de conocer al Hombre» (341).

Al relacionar los textos que hemos visto con el proyecto de Carpentier es preciso cuestionar la originalidad del mismo en la medida que recupera o no una «tradición» latinoamericana. ${ }^{15}$ Algunas de las teorías desarrolladas en el libro La invención de América de Edmundo O'Gorman proporcionarán la estructura para esta discusión, la cual me permitirá explorar más profundamente lo original de Carpentier.

Si bien durante los siglos XV y XVI la realidad de la «cuarta orbis pars»-vacía en términos significativos-se prestaba para su invención europea, en los siglos XIX Y XX, es el producto ideológico de estamismá creación el que genera el proyecto carpentieriano. Mientras que los artistas europeos se apegaron a las expresiones exóticas de lo poco que quedaba de un paraíso perdido, irremediablemente desaparecido, Carpentier logró hallarlo en las expresiones sineréticas que 
son los productos de la gradual simbiosis cultural de hace más de 400 años. Al descubrir un fenómeno tanto europeo como americano para luego crear una estética original y representativa de Latinoamerica, el autor cubano sobrepasa los anhelos de muchos intelectuales europeos quienes no fueron más allá de la simple explotación de la diferencia, es decir «otredad,» de las culturas «primitivas,» $\sin$ percibir la extraña magia de su barroco mestizaje. De esta manera, cuando menos, se distingue Carpentier del grupo de «primitivistas» del siglo pasado que, después de agotar sus recursos estéticos y caer en la falsificación del surrealismo, no aprehendieron, como Carpentier, el significado barroco-sincrético en una expresión original, patrimonio cultural de Latinoamérica.

No obstante, este patrimonio es problemático ya que el concepto de «lo real-maravilloso» se basa en una percepción de la realidad modelada a través de Europa hasta tal grado que constituye lo que no se incorpora en la realidad cotidiana (tanto psicológica como geográfica) de la vida europea. En este sentido, después de haber sido «inventado» en el siglo XV, América y su literatura todavía sirven como una ausencia-es decir, constituyen lo que no es Europa pero también lo que Europa jamás logrará ser (O'Gorman 83). Al basar su estética en términos de una proll:mgada hegemonía cultural, se puede arguir que Carpentier realmente no proporciona ninguna visión que no sea la que se ve a través del ojo europeo y de esta manera continúa la invención de América. Al demostrar la manera en que Latinoamérica no es como Europa, ésta se define y América se queda con el papel de incorporar la sombra de lo que no es la civilización greco-latina. De modo que no sólo fue modelado «lo real-maravilloso» para un .público europeo, sino que también, en gran parte, es producto de la misma cultura occidental; aunque en este caso se ubica fuera del mundo conocido.

En ambos casos, de manera inversa, los dos mundos se descubren a través del otro. De modo que «lo real-maravilloso» de Carpentier incorpora el producto cultural de la conquista europea de una realidad extraordinaria (desconocida) en una articulación ideológica que constituye una concesión a la previa invención y definición europea. Hay que recordar que «lo real-maravilloso» americano sólo 10 es porque se ve a través del ojo europeo y lo que ve es precisamente la realización de su experiencia histórica cristalizada en palacios que recuerdan las cárceles de Piranesi, o regímenes napoleónicos gobernados por negros importados del África.

Las maravillas arquitectónicas que Carpentier atestigua en ]a ciudadela La Ferriere vienen de una «obra sin antecedentes arquitectónicos, únicamente anunciada por las Prisiones imaginarias del Piranese» (16), y también sirven como ejemplos de «lo real-maravilloso» que parece ser, en realidad, el producto de los anhelos europeos concretizados en el Nuevo Mundo. Representa, como ha 
demostrado Edmundo Q'Gonnan, una invención cuya proyección es producto del viejo continente, que simplemente ha utilizado América como repositorio de su fantasías y necesidades más anheladas-una utopía que nunca ha dejado serlo. Al contemplar está situación el historiador mexicano también nos recuerda que América no estará siempre en una posición sumisa en cuanto a su representación ontológica. Señala que:

mientras más se realiza América en su historia al ir actualizando con mayor plenitud la posibilidad original que la constituye, menos propiamente americana es su historia hasta tal punto que dejará de ser... Pero al dotar Europa a América con su ser, inició su propia desintegración ontológica. $(96,98)$

Lo «real-maravilloso,» como lo explica Carpentier:

surge de una inesperada alteración de la realidad (el milagro), de una revelación privilegiada de la realidad, de una iluminación inhabitual o singularmentefavorecedora de las inadvertidas riquezas de la reaLidad, de una ampliación de las escalas y categorías de la realidad, percibidas con particular intensidad en virtud de una exaltación del esp(ritu que lo conduce a un modo de «estado ümite». (15)

En este caso, el milagro sólo existe como reflejo torcido de la realidad (europea) que lo crea y define al considerarlo una alteración de 10 aceptado. No obstante, inmediatamente después habla Carpentier de las «inadvertidas riquezas de la realidad» como una totalidad, evitando-en una simbiosis cultural-la definición eurocéntrica previamente citada. El <aspecto prodigioso de este fenómeno, con sus tonos religiosos (milagro, revelación, iluminación, exaltación, etc.) recuerda el movimiento simbolista, y luego primitivista, al mantenerse en la corriente del arte como filosofía (religión) salvò que, en el caso de Carpentier, se aplica directamente a América donde, según él, tiene sus raíces.

En el prólogo a El reino de este mundo, Carpentier ataca con vehemencia a los surrealistas, encabezados por André Bretón, como embusteros prog'ramados cuyas creaciones no poseen ningún valor artístico porque no son productos de una experiencia real, sino de estados oníricos que en su mayor parte no responden a ninguna realidad vigente. Sin embargo, no se puede olvidar que fue precisamente este movimiento incorporado en el Manifeste du Surrealisme: Poisson soluble, de 1924, lo que instaló en el joven autor cubano una convicción artística que tendría una trascendencia filósofico-estétíca que todavíainfluye en la literatura latinoamericana. Un ejemplo tajante de este desprecio hacia lo que en efecto le hizo comprender el destino de las letras hispanoamericanas, se encuentra en su 
evaluación del pintor frances André Masson en el contexto de la experiencia americana: «Cuando André Masson quiso dibujar la selva de la isla de Martinica, con el increíble entrelazamiento de sus plantas y la obscena promiscuidad de ciertos frutos, la maravillosa verdad del asunto devoró al pintor, dejándolo poco menos que impotente frente al papel en blanco» (14). Como hemos visto, esta situación parece prefigurar la experiencia del narrador de Los pasos perdidos quien se da cuenta que sus puntos de referencia incorporados a las pinturas del Bosco, por ejemplo, no bastan para recrear la realidad tal y como es experimentada en elNuevo Mundo.

Curiosamente, en el mismo prólogo, Carpentier parece admitir cierta deuda a la comunidad artística europea de la época de Cervantes hasta la de Gauguin al subrayar la necesidad de la «fe» para suscitar «lo real-maravilloso.» Para demostrar esto, insiste en que «los que no son Quijotes no pueden meterse, en cuerpo, alma y bienes, en el mundo de Amadís de Gaula o Tirante el Blanco» y que «a Van Gogh [íntimo amigo de Gauguin] le bastaba con tener fe en el Girasol para fijar su revelación en una telas (15). En otras palabras, uno de los requisitos más importantes de la experiencia maravillosa-la «fe»-ha formado parte de la cultura occidental desde Cervantes hasta Van Gogh y, en las palabras de Carpentier, hay que «suscitar» esta experiencia aunque, según él, existe lo maravilloso americano en su estado bruto y sólo hay que recordarlo en todo su esplendor. Obviamente el verbo suscitar implica la noción de crear algo, no necesariamente recordar lo que uno experimenta.

Al considerar la génesis de «lo real-maravilloso» como una negación consciente de los manifestos artísticos de los surrealistas franceses, en particular los de André Breton, por otra parte hay que reconocer que los mismos surrealistas surgieron de un contex to cultural-estético que tiene sus bases en los movimientos artísticos «modernos» incorporados en la obra de Pablo Picasso, André Rousseau y Paul Gauguin, etc. Este último, quien a finales del siglo XIX, intentó resucitar en Europa (Bretaña) lo que Carpentier designaría como la magia de una mitología occidental ya agotada, finalmente la encuentra en las islas de Oceanía. Gauguin prefigura en muchos sentidos la trayectoria artística de Carpentier quien, después de su viaje a Haití en 1943, fOljó su estética para reflejar la gran impresión que le había hecho su contacto con «lo real-maravilloso» americano. No obstante, el hecho de que la añoranza o la necesidad de encontrar aquel mundo mágico haya surgido del contexto europeo (recordemos que Carpentier pasó once años cruciales en París) se parece mucho también a lo que sucedi6-siguiendo las teorías de O'Gorman y Fuentes-en la Europa de Colón y Vespucci cuando apareció una necesidad de encontrar un nuevo mundo-un repositorio de los grandes anhelos espirituales del hombre. 


\section{Notas}

1 La frase «lo real-maravillosow se encuentra entre comillas a lo largo de este artículo para fijar uno de sus múltiples significados. En este caso, empleo la frase para describir un aspecto fundamental del proyecto literario de Alejo Carpentier, según fue articulado por primera vez en el prólogo a la primera edición de El.reino de este mundo y más tarde en su libro de ensayos Tientos y diferencias. En el susodicho prólogo encontramos el primer empleo de esta frase cuando Carpentier describe su viaje a Haití y sus sensaciones «al encontrarme con algo que podríamos llamar lo real-maravilloso» (16). También se encuentra en una formulación más elaborada en Tientos y diferencias. Carpentier señala que «una primera noción de lo real-maravilloso me vino a la mente cuando, a fines del año 1943, tuve la suerte de poder visitar el reino de Henri Christophe ... » (114). Sin embargo, como ha señalado Alexis Márquez Rodríguez en su estudio sobre Carpentier titulado Lo barroco y 10 real-maravilloso en la obra de Alejo Carpentier: «Años más tarde, en una conferencia dictada en la Universidad Centralde Venezuela, en Caracas, amplió y precisó aquella referencia: 'En el año 1943 voy a Haití, casualmente, en compañía del actor Louis Jouvet, y me hallo ahí ante los prodigios de un mundo mágico, de un mundo sincrético, de un mundo donde hallaba al estado vivo, al estado bruto, ya hecho, preparado, mostrado, todo aquello que los surrealistas, hay que decirlo, fabricaban demasiado a menudo a base de artificio ( ... Surge en mí esa percepción de algo que desde entonces no me ha abandonado, que es la percepción de lo que yo llamo lo real-maravilloso, que difiere del realismo mágico y del surrealismo en sí' (40). Por lo tanto me parece legítimo emplear el verbo «concebir» para definir la manera en que surgió el proyecto carpentieriano. Ya que en las tres articulaciones citadas arriba, queda bien claro dónde y cuándo el autor ha fijado el origen de su movimiento literario.

${ }^{2}$ Empleo la frase «la edad moderna» para fijar aquel momento al principio del siglo pasado que-en el mundo artístico—constituláa una separación consciente con el pasado cultural y una búsqueda de nuevas formas expresivas (tanto literarias como plásticas). He elegido la frase «la edad moderna» en vez del r6tulo «modernismo» para evitar confusiones semánticas que,. para el lector latinoamericano, resultan obvias. Sam Hunter y John Jacobus designan el año 1886 como el comienzo del modernismo basándose en la formación, en aquel año. del «Salon des Independants» en París. lo cual fomentaría la creación artística que se ocúpaba de hallar nuevas formas de expresión plástica que iban directamente en contra del arte neo-clásico tan de moda en la Europa del fin de siécle. Uno de los elementos más importantes de este quiebre fue la noci6n de «l'art pour I'art» que libr6 al artista de la verosimilitud serví! del arte contemporáneo y, por consiguiente, le permitía al artista el lujo de reconocer la plasticidad de su materia y crear visiones nunca representadas (Aunter 9-22).

El término utopía es una voz griega con dos posibles interpretaciones que, al cotejarse, crean una juxtaposición de dos circunstancias aparentemente .exclusivas. De un lado, la palabra parece estar compuesta del prefijo griego eu (buen) concatenado a la palabra topos. (lugar) y, por lo tanto, significa un lugar bueno. No obstante, también se puede derivar del prefijo ou (no) más topos (Iugar)-es decir un no-lugar o lugar imposible (Shipley 144). 
$3 \mathrm{El}$ verbo «descubrir» se encuentra entre comillas para indicar la constante presencia de las ideas del historiador mex.icano Edmundo O'Gonnan-presentadas en su libro La invención de América-a lo largo de este trabajo y el reconocimiento de su oposición a esta actitud en cuanto al proyecto de Colón. Pongo ia palabra «primitivo» entre comillas para precisar mi actitud hacia su definición. No se utiliza como término despectivo ya que no constituye la expresión de un mundo ingenuo, bárbaro o no complicado. sino las de una cultura primordial..e incorrupta, libre de los vicios del mundo civilizado que padecen los dos periodos bajo escrutinio aquí. De hecho, el primitivismo se puede definir como la creencia en la superioridad de una vida que se desarrolla dentro de la naturaleza, exenta de la contaminación moral y espiritual de la civilizaci6n. Más tarde veremos estas actitudes expresadas en los escritos de Cristóbal Colón y Paul Gauguin, respectivamente. 4 El continuo apego de Carpentier a su manifesto en forma de prólogo de 1948 es apoyado por el mismo autor quien. al final de su vida, reitera y amplifica estas ideas fundamentales de su proyecto literario. Véase la nota 1 que hace un breve resumen del desarrollo del proyecto carpentieriano y su estabilidad ideológica a lo largo de su vida.

${ }^{5}$ Al poner la palabra «manifiesto entre comiUas quisiera destacar, desde el principio. la íntima relación de «lo reID-maravilloso» con otros movimientos artísticos europeos como el simbolismo, el futurismo. y. claro está, el surrealismo, los cuales aprovecharon este vehículo para diseminar los credos de su proyecto estético-ideológico. Empleo el ténnino ideológico para describir un cuerpo sistemático de conceptos acerca del ser humano y el mundo que habita. De esta manera espero apartarme de una interpretación ajena a mi intención, como sería una lectura sociopolítica (marxista) del ténnino.

6 Basándose en las teorías de Edmundo O'Gorman, Carlos Fuentes señala en cuanto al renacimiento que:

perdidas las estructuras estables del orden medieval, el hombre europeo se siente disminuido y desplazado de su antigua posición central. La tierra se pequeñece en el universo de Copémico. Las pasiones-la voluntad sobre todo-se agrandan para compensar esta disminución. Ambas conmociones se resuelven en el deseo de ensanchar los dominios de la tierra y del hombre: se desea al Nuevo Mundo, se inventa al Nuevo Mundo; se descubre al Nuevo Mundo; se le nombra (... ) La ruptura de la unidad medieval se manifiesta primero en el espacio. Las ciudades amuralladas pierden sus límites, sus contrafuertes se cuartean, sus puentes levadi. zos caen para siempre y a las nuevas ciudades abiertas ... entran atropelladamente las epidemias del escepticismo, el orgullo individual, la ciencia empírica y el crimen contra el Espíritu Santo: las tasas de interés. (50-53)

Más tarde, el autor mexicano ạirma que América proporcionaba una solución para el mundo europeo contemporáneo:

Para el europeo del siglo XVI, el Nuevo Mundo representaba la posibilidad de regeneración del Viejo Mundo. Erasmo y Montaigne, Vives y Moro anuncian el siglo de las guerras religiosas, .uno de los mấs sangrientos de la historia europea, y le contraponen una utopía que finalmente, contradictoriamente, tiene un lugar; América, el espacio del buen salvaje y la edadde oro. ... Recompensar el rostro 
primero del hombre. el rostrofeliz. es la misión del viaje a Utopía: América aparece primero como la Utopía que lavará a Europa de sus pecados históricos" (... ) Europa encuentra en América un espacio que da cabida al exceso de energías del Renacimiento. Pero encuentra también un espacio para limpiar la historia y regenerar el hombre.

En cuanto al arte, también señala que «ha caminado una larga avenida en busca de la tierra feliz de origen, de la isla de Nausica en Homero. a la visita irónica de Luís Buñuel a una isla de esqueletos y excrementos en su película la edad de oro ... » (67) Claro está. uno podría citar una lista inmensa de autores que apoyan o niegan estas teorías. pero yo me limito a invocar a Fuentes, no simplemente porque estoy de acuerdo con sus observaciones, sino también por la sencilla raz6n de que están dirigidas precisamente a nuestro objetivo final; es decir, el concepto de la utopía que sirve como catalizador del discurso creativo.

Son sorprendentes las similitudes hist6ricas entre el siglo XV-XVI y el siglo XIX.XX. Si escrutamos los periodos notamos en primer lugar una inicial euforia y total confianza en los adelantos sociales y científicos del hombre occidental seguido por un periodo de desencanto y desilusión. En el siglo XIX:

al! of Europe simmered with expectations for the future. Many people felt that a new era was dawning in which afuture world would befashioned lo be dramatically and beautifully different from the impeifect world heretofore known. This utopian attitude was pervasive in European intelleetual and artistie cirdesfrom the turn of rhe century through the 1920's and somewhat later in the United States. (Fletcher 16)

Sin embargo, la misma autora advierte que también «one form of socialism that persisted through the Nineteenth Century rejected modem industrialization and urbanization in favor of a simpler life. In what was later to be labeled an anti-utopian view, many people feh that urban and technological development destroyed humanist values and lifestyles» (Fletcher 19). Es en esta corriente que quisiera insertar al pintor Paul Gauguin quien era líder de un grupo que despreciaba la ciudad y su enajenación y proponía la vida «primitiva» de Bretaña y luego del mundo exótico de Tahití corno remedio para este mal. 7 Alexis Márquez Rodríguez, «Alejo Carpentier, punto de partida de La nueva narrativa latinoamericana.» Revista de Estudios Hispánicos. Universidad de Puerto Rico (1983, 9-15).

$8 \mathrm{El}$ «otro» se encuentra entre comillas para precisar su definición ya que el término está ahora muy de moda en círculos académicos (ef Tzvetan Todorov). Aquí denomina a los habitantes de un mundo desconocido (en el siglo XV) o no comprendido (en el siglo XIX) por el hombre europeo. .

${ }^{9}$ Estas ideas en cuanto a la superioridad moral de las culturas de América, aunque primero articuladas por Col6n, son desarrolladas por los humanistas Michel Montaigne (en sus ensayos «De coches» y «De caníbales»), Tomás Moro (Utopía), Bartolomé de las Casas (Brevísima relacion de la destrucción de las indias), entre otros. Estoy endeudado con la doctora Verónica Cortínez por esta observación que da más sustancia a uno de los 
argumentos fundamentales de este trabajo: la percibida inferioridad del mundo occidental y la superioridad del Mundo Nuevo.

b Obviamente el texto de Colón no constituye el único ni el más completo archivo de infonnaci6n sobre las injusticias y barbaridades de la Europa contemporánea. No obstante. es excepcional por la manera en que utiliza la cultura de América para definir y critlcar la suya.

11 La palabra «civilizada» se encuentra entre comillas para hacer destacar la ironía de su empleo para describir muchos de los aspectos de la vida europea de los siglos XIX-XX, es decir. la Europa de la edad moderna.

12 En su libro Modernismo 1890-1930. M. Bradbury y James McFarlane señalan que el modernismo: «is the one art that responds to the scenario of our chaos. It is the art consequent oo Heisenberg's 'Uncertainty principIe,' oCthe destruction oícivilízation and the reason in the First World War. ofthe world changed and ioterpreted by Marx, Freud, and Darwin. of capital and constant industrial acceleration, oí existential exposure to meaningless or absurdity» (27).

13 John Hughes ha observado que Picasso demuestra con este cuadro que: «the tradition of the human figure, which had been the very spine of Western art for two and a half millenia. had at last ron out; and that in order lo renew il8 vitality, one had to look toward untapped cultural resources-the Africans. remote in their othemess.... No painting ever looked so convulsive. None signalled a faster change in the history of arts (21). En su libro Modern Art, Sam Hunter y John Jacobus observan que: «Modernist art received a climactic impulse in 1907 when Picasso marshaled his phenomenal energies and launched upon an enonnously famous, revolutionary picture known as Les Demoiselles d'Avignons" (136). También señalan que «the rịchly contradictory Demoiselles began as a metaphoric statement of good and evil and then became magically transfonned into one oí our most infiuential modemist icons, and a climactic if transitional example oí the expressive force and depth ofPicasso's art ( ... ) This unique combination of formal and psychological factors anticipated important aspects oí both abstract and Surrealist art of the new century» (138).

${ }_{14}$ La versión original de la carta no se encuentra en el libro citado por Herschel B. Chipp. ${ }^{15}$ Con «tradición» se entiende una experiencia histórico-cultural única cuya existencia proporciona un vehículo para el desarrollo de las artes propias de una región.

\section{Obras citadas}

Bradbury, Malcolm, James McFarlane. eds. 1986. Modernism: 1890-1930. Middlesex: Penguin Books Ltd.

Carpentier, Alejo. 1989. Obras Completas. 2 vols. México: Siglo XXI.

Chipp, Herschel B. 1968. Theories ofModernArt: A Source Book by Artists and Critics. Berkely, Los Ángeles: U of California P.

Col6n. Cristóbal. 1986. Los cuatro viajes del almirante y su testamento. Ed. Consuelo Varela. Madrid: Alianza Editorial.

1893. The Spanish letter 01 Columbus to Luis de Sant'Angel. London: Bemard Quaritch. 
Fletcher, Valerie 1. 1983. Dreams and Nightmares: Utopian Vissions in Modern Art. Washington: Smithsonian Institute Press.

Fuentes. Carlos. Valiente mundo nuevo; tpica. utopia y mito en la novela hispanoamericana. Mexico: Fondo de Cultura Económica.

Gold water, Robert J. 1938. Primitivism in Modern Painting . New York: Harper \& Brother Publishers.

Hughes, Robert. 1981. The Shock afthe New. New York: Alfred A. Knopf.

Hunter, Sam. John Jacobus. 1985. Modern Art: Painting. Seulpture. Architecture. New York: Harry N. Abrams, Ine.

Márquez Rodríguez. Alexis. 1984. Lo barroco y lo real-maravilloso en la obra de Alejo Carpemier. Mexico: Siglo XXI Editores.

O'Gorman, Edmundo. 1976. La invención de América. México: Fondo de Cultura Económica.

Shipley, Joseph. 1955. Dictionary of Word Origins. New York: Philosophical Library. 